\section{Gehäuft aggressiver heller Hautkrebs nach Organtransplantation}

Immunsupprimierte haben nicht nur ein erhöhtes Risiko für Nicht-MelanomHauttumoren, auch das Risiko für aggressiv subklinisch expandierende Formen ist gesteigert - vor allem nach einer Organtransplantation.

$\mathrm{B}_{\mathrm{r}}^{\mathrm{e}}$ ei Nicht-Melanom-Karzinomen der Haut ist die sichtbare Läsion mitunter nur die Spitze des Eisbergs, ein Teil der Tumoren infiltriert aggressiv subklinisch das benachbarte Gewebe. Immunsupprimierte Patienten könnten davon besonders betroffen sein, allerdings wurde dies bislang nicht klar nachgewiesen. Bekannt ist jedoch, dass immunsupprimierte Personen insgesamt ein erhöhtes Risiko für weißen Hautkrebs haben. Mit einer retrospektiven Analyse wollten Forscher aus den USA feststellen, ob die Tumoren von solchen Patienten auch gehäuft subklinisch expandieren.

Für die Analyse werteten die Dermatologen Angaben zu knapp 3.000 Mohsschen Exzisionen von Nicht-MelanomTumoren aus. 347 der Patienten (11,5\%) waren immunsupprimiert, bei knapp der Hälfte von ihnen war eine Organtransplantation der Grund, rund ein Drittel hatte HIV und die übrigen litten an anderen Immundefekten. Eine aggressive subklinische Expansion (ASE) wurde bei 805 - also über einem Viertel - der Patienten festgestellt. Von einer ASE gingen die Dermatologen aus, wenn die Chirurgen bei der Mohsschen Operation mindestens zweimal nachschneiden mussten, weil die Ränder infiltriert waren. Zudem sollte der Exzisionsrand zum Schluss mindestens $10 \mathrm{~mm}$ betragen.

Von den ASE-Patienten waren 17\% immunsupprimiert, bei denen ohne ASE lag der Anteil nur bei 10\%. Umgekehrt hatten knapp $40 \%$ der Immunsupprimierten ein ASE-Tumor, aber nur $25 \%$ der Immunkompetenten. Der Anteil von ASE-Patienten zu solchen ohne ASE war bei den Immunsupprimierten rund doppelt so hoch wie bei denen ohne immunologische Nachteile (Odds Ratio, OR = 1,9). Dies ließ sich vor allem auf die hohe ASE-Rate bei Patienten mit Organtransplantation zurückführen $(\mathrm{OR}=2,7)$. Dagegen war diese Rate bei HIV-Patienten $(\mathrm{OR}=1,1)$ nicht und bei hämatologisch Erkrankten nur knapp signifikant erhöht $(\mathrm{OR}=1,7)$. Als weitere ASE-Risikofaktoren erwiesen sich eine Tumorlokalisation im Bereich von Augenbrauen, Lippen und Nase. Basalzellkarzinome dehnten sich ebenfalls häufiger subklinisch aus als Plattenepithelzelltumoren.

Fazit: Immunsupprimierten Patienten mit hellem Hautkrebs haben ein erhöhtes Risiko für ASE-Formen. Chirurgen sollten sich bei diesen Patietnen daher nicht auf die üblichen Exzisionsränder verlassen, so die Autoren.

Thomas Müller

Song SS et al. Nonmelanoma Skin Cancer With Aggressive Subclinical Extension in Immunosuppressed Patients. JAMA Dermatol 2016; 152: 683-90

\title{
Ein Amalgam-Tattoo unter der Zunge
}

Eine 49-jährige Frau ohne Familienanamnese für ein malignes Melanom stellte sich mit einer asymptomatischen hyperpigmentierten Schleimhautveränderung an der Unterseite der Zunge vor. Die Läsion war ihr vor etwa vier Monaten aufgefallen. Die Kollegen dachten zuerst an ein malignes Melanom. Ein Trauma oder vermehrter Alkohol- oder Nikotingenuss lagen nicht vor. Jedoch erzählte die Patientin, dass sie drei Monate bevor die Veränderung auftrat, eine Zahnbehandlung über sich ergehen lassen musste. Die Veränderung an der Unterseite der Zunge war asymmetrisch und braungrau pigmentiert. Zentral hatte man den Eindruck einer kleinen Aufhellung (Abb. A). Andere Haut- oder Schleimhautveränderungen fanden sich nicht. Die Läsion wurde komplett exzi- diert. Histologisch erkannte man Dermis mit einer Fibrose und interstitiellen Ablagerungen eines schwarzen Pigments ohne entzündliches Infiltrat (Abb. B, HE-Färbung). Hinweise auf eine Hyperplasie von Melanozyten oder ein Malignom fanden sich nicht.

Unter der Diagnose einer Amalgam-Tätowierung folgten keine weiteren Maßnahmen. Ein Jahr später war kein Rezidiv aufgetreten. Bei der Vielzahl von Amalgam-Plomben, die früher eingebracht wurden, wundert man sich, dass derartige Veränderungen nicht häufiger beobachtet wurden. Prof. Hermann S. FüeßI

Vera-Kellet $\mathrm{C}$ et al. Oral amalgam tattoo mimicking melanoma. $\mathrm{N}$ Engl J Med 2016; 347: e21
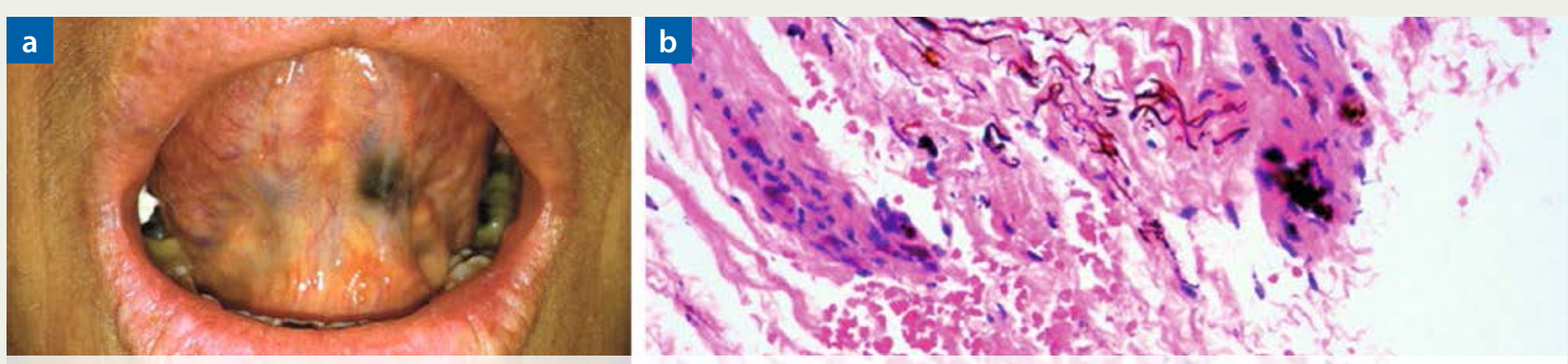

Hyperpigmentierte Schleimhautveränderung an der Unterseite der Zunge (A), Dermis mit interstitiellen Ablagerungen eines schwarzen Pigments (B). 